(C) 1996 IEEE. Personal use of this material is permitted. However, permission to reprint/republish this material

for advertising or promotional purposes or for creating new collective works for resale or redistribution to servers

or lists, or to reuse any copyrighted component of this work in other works must be obtained from the IEEE.

\title{
Implementation Status of the Global and Local Beam Position Feedback Systems for the Advanced Photon Source Storage Ring*
}

\author{
Y. Chung, D. Barr, G. Decker, J. Galayda, J. Kirchman, F. Lenkszus, A. Lumpkin and A. J. Votaw \\ Argonne National Laboratory, Argonne, IL 60439
}

\begin{abstract}
The Advanced Photon Source (APS) is implementing an extensive beam position feedback system for both global and local stabilization of particle and photon beams based on digital signal processing. The description and operational experience of the system will be given in this paper. In particular, we will discuss the underlying fundamental principles, hardware layout, controls interface, and automatic software generation for multiple digital signal processors (DSPs) distributed in $20 \mathrm{VME}$ crates around the ring. The feedback system runs at 4-kHz sampling frequency in order to achieve the correction bandwidth of approximately $100 \mathrm{~Hz}$. For the maximum correction efficiency and resolution of conflicts among multiple local feedback systems due to the local bump closure error, the global and local feedback systems are combined into a single unified system. This novel approach is made possible through data sharing among the global and local systems via the fiber-optically networked reflective memories.
\end{abstract}

\section{INTRODUCTION}

The Advanced Photon Source (APS) is one of the thirdgeneration synchrotron light sources which are characterized by low emittance of the charged particle beams and high brightness of the photon beams radiated from insertion devices (IDs). In order to take the full advantage of intense synchrotron radiation, the incident intensity, position and angle of the x-ray beam need to be tightly controlled [1-3]. Even though every effort is made to stabilize the electrical and mechanical components of the ring, there will inevitably be residual beam motion primarily caused by the quadrupole vibration.

The sources of vibration include ground vibration, mechanical vibration of the accelerator subcomponents, thermal effects, and so forth. These are manifested in the undesired particle and x-ray beam motion. This results in increased beam size and diluted beam emittance in the short term. An example of the long-term effect is the diurnal changes in the ring circumference and periodic shift of the x-ray beam at the user station [4]. At the APS, the stringent transverse $x$-ray beam position stability required by the current user community will be achieved through extensive beam position feedback systems with the correction bandwidth exceeding $100 \mathrm{~Hz}[5]$.

*Work supported by the U.S. Department of Energy, Office of Basic Energy Sciences, under Contract No. W-31-109-ENG-38.
The APS has $360 \mathrm{rf}$ beam position monitors (BPMs) and 318 corrector magnets distributed around the storage ring, miniature BPMs for ID beamlines, and x-ray BPMs in the front end of x-ray beamlines for global and local orbit feedback. The real-time (AC) feedback systems, which are the main focus of this work, will use a subset of these.

The feedback systems can be largely divided into the global and local feedback systems according to the scope of correction. The global feedback system uses $40 \mathrm{rf}$ BPMs and 38 corrector magnets distributed in 40 sectors. The primary function is to stabilize the selected perturbation modes of the global orbit. The local feedback systems, on the other hand, stabilize the source regions of the $\mathrm{x}$-ray beams locally for angle and displacement.

An ideal local feedback system would not affect the rest of the closed orbit including other local feedback systems. In reality, the global and local feedback systems constantly interact with one another. The effect of global orbit feedback unavoidably interferes with the local feedback. On the other hand, the bump closure error in the local feedback due to bump coefficient error, magnet field error, eddy current effect, etc., causes global orbit perturbation and affects other local feedback systems. If this interaction is too strong, the feedback systems can become ineffective, oscillatory, or even unstable. In order to minimize such effects and maximize the feedback efficiency, it is necessary to decouple the global and local feedback systems.

In this work, we will report on the current status of implementation of the APS orbit feedback systems. The remainder of this paper will be an overview of the feedback algorithm and system description in Section II, system performance test results in Section III, and the current status of system integration in Section IV.

\section{FEEDBACK ALGORITHM AND SYSTEM DESCRIPTION}

The APS orbit feedback system has the capability to handle multiple local feedback systems for control of the insertion device (ID) and bending magnet (BM) $\mathrm{x}$-ray beamlines and a global feedback system to minimize the global orbit distortion and thus to maintain high beam quality. In this section, we will describe the algorithms governing the operation of these feedback systems [5].

\section{A. Control Algorithm}

The signal processing core of the feedback systems is based on digital signal processing (DSP) [6]. The design sampling frequency or the number of feedback loops executed 
per second is $4 \mathrm{kHz}$. Figure 1 shows the schematic of a multichannel digital feedback system. The object to be controlled, or the plant, is represented by the matrix $\mathbf{R} . \mathbf{R}_{i n v}$ is the inverse-model matrix that controls coupling of various control points in the plant. In the case of orbit feedback, $\mathbf{R}$ is a composition of the global and local response matrices.

The gain matrix $\mathbf{G}$ comprises the low pass filter (LPF); proportional, integral, and derivative (PID) controller; and any compensation filter (CF) that cancels the undesirable frequency dependence in the plant, such as the effect of the eddy current in the vacuum chamber. $\mathbf{H}$ represents the BPMs.

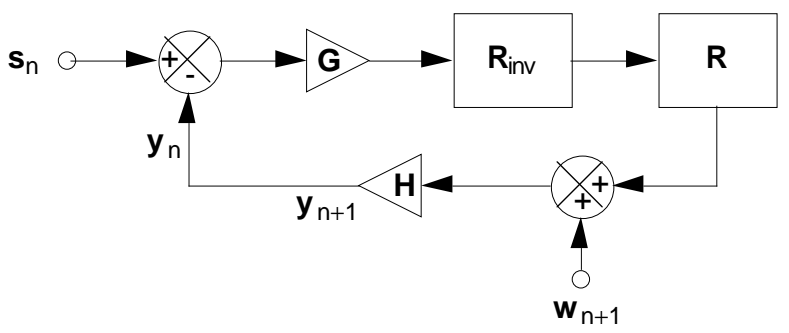

Fig. 1: The schematic diagram of a digital feedback system.

\section{B. Global Orbit Feedback}

The global feedback attenuates global orbit distortion by using 40 BPMs and 38 correctors distributed around the ring. One BPM and one corrector per each sector are used, except for sectors 39 and 40 where the correctors were not installed due to space limitation near the injection point. The vacuum chamber at the location of the global corrector is made of thin stainless steel unlike the local feedback systems.

The DSPs and interface boards are installed in twenty VME crates distributed around the ring. Each crate covers two sectors for both the horizontal and vertical planes, an odd sector in the upstream and an even sector in the downstream. A single DSP is assigned per four degrees of freedom.

Since each DSP needs access to the global orbit data at 40 global BPMs, data collected from all 40 sectors needs to be available across the local VME bus. This is done via a dedicated fiber optic network of reflective memories with a data transfer rate of $26 \mathrm{Mbytes} / \mathrm{sec}$. For each degree of freedom, the 40 BPM data are multiplied by a row of the global inverse response matrix obtained using the singular value decomposition (SVD) technique $[3,5,7]$. Operation of the DSPs is synchronized by an event signal broadcasted from a central $4-\mathrm{kHz}$ clock.

\section{Local Orbit Feedback}

A local orbit feedback system is based on a four-magnet local bump. The beam positions are detected by two rf BPMs inside the local bump and two X-ray BPMs [2]. This enables control of both the angle and displacement of the X-ray beam.

Four-magnet local bumps can be decomposed into two independent three-magnet local bumps, $a$ and $b$, and transformaion between the bump strengths and the beam positions is straightforward. The locality of the bump can be achieved by powering the bump magnets in certain ratios determined by local bump coefficients. Empirical derivation of these coefficients is discussed in [8]. The local response matrix $\mathbf{R}_{l}$ can then be reduced to a $2 \times 2$ matrix relating two beam positions and two bump strengths.

Imbalance in the bump coefficients can cause local bump closure error and introduce global orbit distortion. Even though the coefficients are well matched at DC, eddy current effect in the relatively thick (1/2") aluminum vacuum chamber will cause significant bump closure error if orbit perturbation contains components with high enough frequency. This is remedied by global feedback.

\section{Decoupling of Global and Local Feedback Systems}

In the ideal situation of zero local bump closure error, the local feedback systems are transparent to the rest of the ring. However, the global feedback unavoidably interferes with the operation of the local feedback systems unless the source is localized and does not cause global orbit distortion. In general, orbit perturbation is seen by both the global and local feedback systems, and they will attempt to correct it independently. This can be resolved by decoupling the global and local feedback systems [5]. This unified approach in effect combines the global and local feedback systems, renders the entire system into multiple non-interacting feedback systems, and thus minimizes the interference.

This scheme requires that the local feedback systems take into account the global orbit distortion as well as the local orbit error. This is readily met since the global orbit data is available in all VME crates through the reflective memories.

\section{E. Hardware/Software Configuration and User Interface}

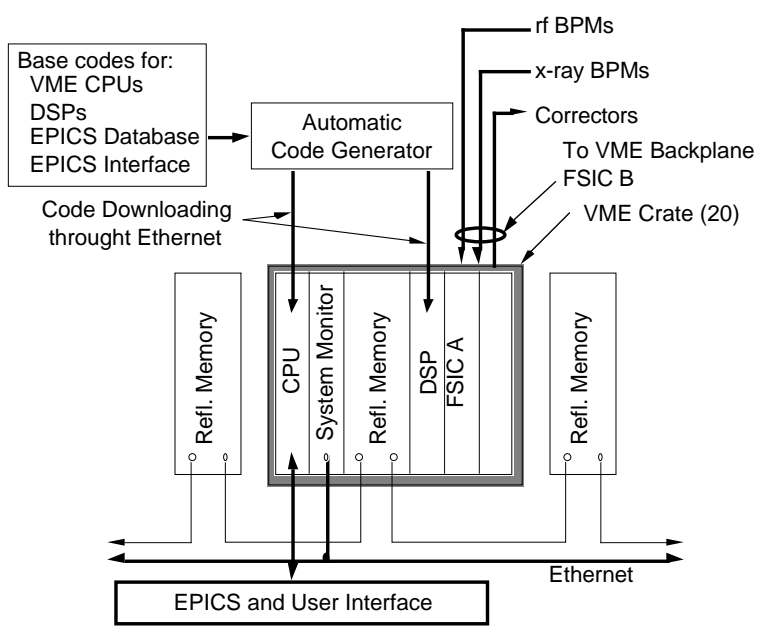

Fig. 2: Operations interface to the orbit feedback system.

Figure 2 shows the operations interface to an orbit feedback system crate. There are a total of 20 such crates, each of which has one VME CPU and multiple DSP boards. Considering the number of processors in the system, it would be impractical and error-prone to write, compile, and link individual codes for each processor. Instead, the codes are generated automatically from base source codes which contain instructions for code generation for different processors. The 
command parser is based on the GUS kernel, a script-based interpretive shell developed for streamlined data acquisition and analysis [9]. The compiled codes for the VME CPUs, DSPs, EPICS (Experimental Physics and Industrial Control System) data base, and controls interface are downloaded to the target processors through the ethernet connection. Once the codes begin execution in the processors, the feedback operation, including data sampling and analysis, can be controlled via EPICS and the user interface.

The rf BPMs, x-ray BPMs, and corrector power supplies are interfaced through Feedback System Interface Controller A (FSIC A) and the sisterboard FSIC B in the VME backplane. Reflective memories are used for global orbit data sharing and are networked through fiber optic cables. During feedback operation, the DSPs perform direct data I/O with FSIC A and reflective memory through the local VME bus.

\section{SYSTEM PERFORMANCE}

The performance of the global feedback system is shown in Fig. 3 in the time and frequency domains using the sampling frequency of $4 \mathrm{kHz}$, LPF bandwidth of $50 \mathrm{~Hz}$, and the PID gains $\mathrm{K}_{\mathrm{p}}=10, \mathrm{~K}_{\mathrm{i}}=0.5$, and $\mathrm{K}_{\mathrm{d}}=2$. For both tests, the global orbit perturbation was simulated using a single steering error in the ring. In the time domain, the steering error introduced a step impulse at $t=10 \mathrm{~ms}$. Comparison of the orbit motion with feedback on and off is shown Fig. 3(a). The response
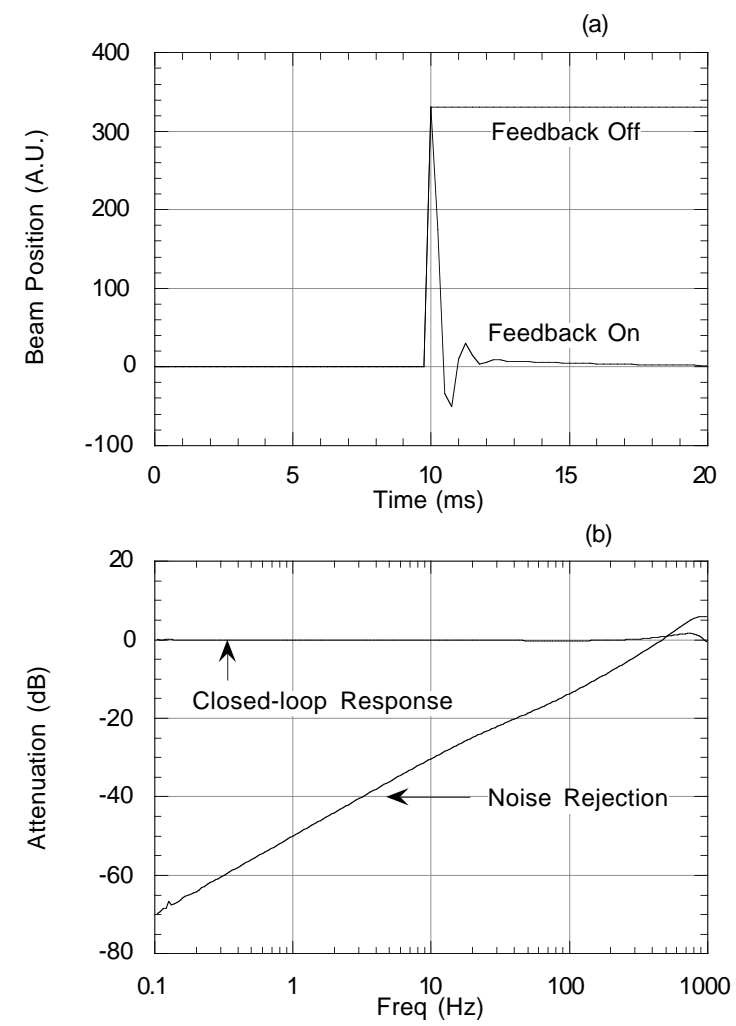

Fig. 3: (a) Impulse response of the global feedback system. (b) Frequency response. The sampling frequency was $4 \mathrm{kHz}$, LPF bandwidth was $50 \mathrm{~Hz}$, and the PID gains were: $\mathrm{K}_{\mathrm{p}}=10$, $\mathrm{K}_{\mathrm{i}}=0.5$, and $\mathrm{K}_{\mathrm{d}}=2$. time is approximately $500 \mu \mathrm{s}$. The system performance in the frequency domain is shown in Fig. 3(b). The system bandwidth at $-6 \mathrm{~dB}$ noise rejection is $250 \mathrm{~Hz}$. Similar performance results have been obtained from the local feedback system test on the ESRF storage ring [8].

\section{CURRENT STATUS}

At this time, the backbone hardware, including the VME crates, VME CPUs, DSPs, reflective memories, and communication network, has been installed and tested. Automatic code generator has been developed to facilitate compiling and downloading of the software for the VME CPUs and DSPs. The performance of the integrated system indicates response time of $500 \mu$ s and correction bandwidth of $250 \mathrm{~Hz}$ at $-6 \mathrm{~dB}$ noise rejection.

The APS storage ring is currently in the commissioning phase. As the ring performance stabilizes in the near future, one or two local feedback systems will be installed on selected $\mathrm{x}$-ray beamlines. With completion of production of the BPM and corrector power supply interface boards expected this fall, the global feedback system will be implemented.

\section{REFERENCES}

[1] R. Hettel, "Beam Steering at the Stanford Synchrotron Radiation Laboratory," IEEE Trans. Nucl. Sci., NS-30, No. 4, p. 2228, 1983.

[2] R. J. Nawrocky et al., "Automatic Beam Steering in the NSLS Storage Rings Using Closed Orbit Feedback," Nucl. Instr. and Meth., A266, p. 164, 1988.

[3] Y. Chung, et al., "Closed Orbit Feedback with Digital Signal Processing," Proceedings of the 1994 European Particle Accelerator Conference, London, U.K., p. 1592, 1994.

[4] R. Hettel, et al., "Design and Characterization of the SSRL Orbit Feedback System," Proceedings of the 1994 European Particle Accelerator Conference, London, U.K., p. 1580, 1994.

[5] Y. Chung, "A Unified Approach to Global and Local Beam Position Feedback," Proceedings of the 1994 European Particle Accelerator Conference, London, U.K., p. 1595, 1994.

[6] A. Peled and B. Liu, Digital Signal Processing, (John Wiley \& Sons, 1976).

[7] Y. Chung, G. Decker and K. Evans, Jr., "Closed Orbit Correction Using Singular Value Decomposition of the Response Matrix," Proceedings of the 1993 IEEE Particle Accelerator Conference, Washington, D.C., p. 2263, 1993.

[8] Y. Chung, et al., "Local Beam Position Feedback Experiments on the ESRF Storage Ring," these proceedings.

[9] Y. Chung, et al., "Development of GUS for Control Applications at the Advanced Photon Source," Proceedings of the 1994 European Particle Accelerator Conference, London, U.K., p. 1794, 1994. 